\title{
Plastic debris distribution and behaviour in soil: new key insights from a household wastes- contaminated soil.
}

\author{
AURÉLIE WAHL ${ }^{1}$, MELANIE DAVRANCHE ${ }^{2}$ AND \\ JULIEN GIGAULT ${ }^{3}$ \\ ${ }^{1}$ Univ. Rennes, CNRS, Geosciences Rennes, UMR 6118 \\ ${ }^{2}$ Univ Rennes, CNRS, Géosciences Rennes, UMR 6118 \\ ${ }^{3}$ University of Laval \\ Presenting Author: aurelie.wahl@univ-rennes1.fr
}

Plastic contamination is widely acknowledged as one of the major environmental and human health threats. Yet, from all environmental compartments investigated, terrestrial ecosystems, especially soils, remain poorly studied. However, considering the plastic debris budget, global inputs to soils may intuitively be greater than the ones entering into oceans. Better understanding their fate and behaviour in soils becomes therefore crucial and urgent.

While the detection and distribution of microplastic in soils is improving, we have just demonstrated the nanoplastics occurrence. For this purpose, we investigated a soil contaminated with plastic debris from household wastes. To address the increasing volume of urban waste, household wastes were composted for agricultural purposes thirty years ago. However, due to a mismanagement, a large amount of plastics was crushed, composted and spread on agricultural lands. These contaminated soils can be considered as a proxy to investigate the plastics degradation as well as their fate and behaviour under natural soil conditions. Based on this site, we developed the first methodology to extract, characterise and identify nanoplastics in such a complex system. Then, as microplastics are visually identified through the soil profile, what about the nanoplastics? Are they leached to groundwater? We thus compared the nanoplastics occurrence to that of microplastics. This new extraction method was then applied to a soil profile while simultaneously, microplastics from 2 to $5 \mathrm{~mm}$ were extracted by sieving and density fractionation. The microplastic amount in the upper most soil horizons was higher than in the deeper. This microplastic distribution was compared to the nanoplastics presence to estimate their potential of transfer in soil. Finally, both micro- and nanoplastics compositions were compared.

These results, based on both micro- and nanoplastics composition and distribution, represent the first insights on plastic debris fate and behaviour under soil conditions and allow rising both fundamental knowledge on emerging processes affecting soils and new consideration and perspectives for soil protection as well. 